PROCEEDINGS OF THE

AMERICAN MATHEMATICAL SOCIETY

Volume 136, Number 11, November 2008, Pages 4079-4087

S 0002-9939(08)09372-6

Article electronically published on June 4, 2008

\title{
POLYNOMIAL SPLITTINGS OF METABELIAN VON NEUMANN RHO-INVARIANTS OF KNOTS
}

\author{
SE-GOO KIM AND TAEHEE KIM
}

(Communicated by Daniel Ruberman)

\begin{abstract}
We show that if the connected sum of two knots with coprime Alexander polynomials has vanishing von Neumann $\rho$-invariants associated with certain metabelian representations, then so do both knots. As an application, we give a new example of an infinite family of knots which are linearly independent in the knot concordance group.
\end{abstract}

\section{INTRODUCTION}

A knot $K$ in the 3 -sphere $S^{3}$ is said to be slice if there is a locally flat 2-disk $D$ embedded in the 4 -ball $B^{4}$ with $\partial\left(B^{4}, D\right)=\left(S^{3}, K\right)$. A pair of knots $K_{1}$ and $K_{2}$ are concordant if $K_{1} \#\left(-K_{2}\right)$ is slice where $-K$ is the mirror image of $K$ with reversed orientation. The set of concordance classes of knots forms an abelian group under connected sum, called the knot concordance group and denoted by $\mathcal{C}$. In $\mathcal{C}$ the identity 0 is represented by slice knots.

By constructing sliceness obstructions using Seifert forms, Levine showed that the knot concordance group surjects to $\mathbb{Z}^{\infty} \oplus(\mathbb{Z} / 2)^{\infty} \oplus(\mathbb{Z} / 4)^{\infty}$ [13, 14]. A knot with vanishing Levine obstructions is called algebraically slice. Using their own invariants, Casson and Gordon showed that there are nonslice knots which are algebraically slice [1. Using Casson-Gordon invariants Jiang showed that the concordance group of algebraically slice knots is infinitely generated 9]. Gilmer refined Casson-Gordon invariants by combining Casson-Gordon invariants with the Levine obstructions 7 .

In [4] Cochran, Orr and Teichner made progress by establishing a geometric filtration of $\mathcal{C}$,

$$
0 \subset \cdots \subset \mathcal{F}_{n .5} \subset \mathcal{F}_{n} \subset \cdots \subset \mathcal{F}_{1.5} \subset \mathcal{F}_{1} \subset \mathcal{F}_{0.5} \subset \mathcal{F}_{0} \subset \mathcal{C},
$$

where the subgroup $\mathcal{F}_{h}$ is the set of all $(h)$-solvable knots. They showed that this filtration is closely related to the known concordance invariants. For instance, a knot lies in $\mathcal{F}_{0.5}$ if and only if the knot is algebraically slice [4, Remark 1.3.2]. Also they proved that all previously known concordance invariants vanish on (1.5)solvable knots [4, Section 9]. In particular, Casson-Gordon-Gilmer invariants [1, 7] vanish on (1.5)-solvable knots. Cochran, Orr and Teichner [4, 5] used von Neumann $\rho$-invariants $\left(L^{2}\right.$-signature defects) to prove that the quotient group $\mathcal{F}_{2} / \mathcal{F}_{2.5}$

Received by the editors May 11, 2007, and, in revised form, October 8, 2007. 2000 Mathematics Subject Classification. Primary 57M25; Secondary 57N70.

Key words and phrases. Knot, concordance, polynomial splitting. 
has infinite rank. The second author [11] proved that $\mathcal{F}_{1} / \mathcal{F}_{1.5}$ has an infinite rank subgroup of knots for which Casson-Gordon invariants vanish. In their respective papers Cochran-Orr-Teichner [4] and the second author [1] constructed their examples using genetic modification and showed the linear independence using signature functions whose integrals over $S^{1}$ are linearly independent.

Linear independence of knots in $\mathcal{C}$ may be checked in a different manner using relative primeness of Alexander polynomials. Such an approach was first given by Levine [13, who showed that if the connected sum of two knots with coprime Alexander polynomials has vanishing Levine obstructions, then so do both knots. The first author [10] showed that the Casson-Gordon-Gilmer invariants split in this way as well.

In this paper, we prove a similar splitting property for the von Neumann $\rho$ invariants of knots associated with certain metabelian representations. Let $K$ be a knot and $M_{K}$ the zero surgery on the knot $K$ in $S^{3}$. Let $\Lambda:=\mathbb{Q}\left[t^{ \pm 1}\right]$. For every $x$ in the (rational) Alexander module $H_{1}\left(M_{K} ; \Lambda\right)$, one can obtain the real-valued $\rho$-invariant $\rho\left(K, \phi_{x}\right)$ where $\phi_{x}: \pi_{1}\left(M_{K}\right) \rightarrow \mathbb{Q}(t) / \Lambda \rtimes \mathbb{Z}$ is the homomorphism associated with $x$ via the Blanchfield linking form (see Definition 2.3). We say that $K$ has vanishing $\rho$-invariants if there exists a self-annihilating $\Lambda$-submodule $P$ of $H_{1}\left(M_{K} ; \Lambda\right)$ with respect to the Blanchfield linking form (hence algebraically slice) such that $\rho\left(K, \phi_{x}\right)=0$ for all $x \in P$. For the definition of self-annihilating submodule, see Section 2. Cochran, Orr and Teichner showed that (1.5)-solvable knots have vanishing $\rho$-invariants [4, Theorem 4.6]. This yields a sliceness obstruction since a slice knot is (1.5)-solvable.

We state the main theorem:

Theorem 1.1. Let $K_{1}$ and $K_{2}$ be knots with coprime Alexander polynomials. If $K_{1} \# K_{2}$ has vanishing $\rho$-invariants, then so do both $K_{1}$ and $K_{2}$.

We give a stronger form of this theorem in Theorem 3.1.

To demonstrate the strength of this result, in Section 4 we give a new example of infinitely many knots with vanishing Casson-Gordon invariants which are linearly independent in $\mathcal{F}_{1} / \mathcal{F}_{1.5}$ and hence in $\mathcal{C}$.

\section{Preliminaries}

In this section we briefly review the machinery necessary for this paper. In [4, Cochran, Orr and Teichner established a filtration $\left\{\mathcal{F}_{h}\right\}_{h \in \frac{1}{2} \mathbb{N}_{0}}$ of $\mathcal{C}$ indexed by nonnegative half-integers where $\mathcal{F}_{h}$ is the subgroup ofdefinition $(h)$-solvable knots defined below. Recall that for a group $G$ and a nonnegative integer $n$, the $n$-th derived group of $G, G^{(n)}$, is defined inductively by the relations $G^{(0)}:=G$ and $G^{(k)}:=\left[G^{(k-1)}, G^{(k-1)}\right]$ for $k \geq 1$.

For a $\mathrm{CW}$-complex $W$, we define $W^{(n)}$ to be the regular covering corresponding to the subgroup $\left(\pi_{1}(W)\right)^{(n)}$. Suppose $W$ is an oriented $4-$ manifold. Then there is an intersection form

$$
\lambda_{n}: H_{2}\left(W^{(n)}\right) \times H_{2}\left(W^{(n)}\right) \rightarrow \mathbb{Z}\left[\pi_{1}(W) / \pi_{1}(W)^{(n)}\right] .
$$

Also there is a self-intersection form $\mu_{n}$ on $H_{2}\left(W^{(n)}\right)$. For more details about these forms refer to [17] and [4, Section 7]. For a nonnegative integer $n$, an $(n)-$ Lagrangian is a submodule $L \subset H_{2}\left(W^{(n)}\right)$ on which $\lambda_{n}$ and $\mu_{n}$ vanish and which 
maps onto a Lagrangian of $\lambda_{0}$ under the homomorphism induced by the covering map.

Definition 2.1 ([4, Section 8]). Let $n \in \mathbb{N}_{0}$. A knot $K$ is called $(n)$-solvable if $M_{K}$ bounds a spin 4-manifold $W$ such that the inclusion map $M_{K} \rightarrow W$ induces an isomorphism on the first homology and such that $W$ admits an $(n)$-Lagrangian with $(n)$-duals. This means that the intersection form $\lambda_{n}$ pairs the $(n)$-Lagrangian and the $(n)$-duals nonsingularly and that their images together freely generate $H_{2}(W)$. The 4 -manifold $W$ is called an $(n)$-solution for $K$, and we say $K$ is $(n)$-solvable via $W$.

Similarly, we define (n.5)-solvable knots for $n \in \mathbb{N}_{0}$ in such a way that an (n.5)solution $W$ is required to admit an $(n+1)$-Lagrangian with $(n)$-duals. For more details, refer to [4, Definitions 8.5 and 8.7].

Cochran, Orr and Teichner showed that every slice knot is $(h)$-solvable for all nonnegative half-integers $h$ [4, Remark 1.3.1]. They detect (n.5)-solvable knots using the von Neumann $\rho$-invariants [4, Theorem 4.2].

Although the von Neumann $\rho$-invariants are defined in a more general setting, for our purpose, henceforth we study the von Neumann $\rho$-invariants associated with the representations to the metabelian group $\Gamma:=\mathbb{Q}(t) / \Lambda \rtimes \mathbb{Z}$ where $\mathbb{Z}$ is generated by $t$ acting on $\mathbb{Q}(t) / \Lambda$ by multiplication. In [4 the group $\Gamma$ is called the first rationally universal group and is denoted by $\Gamma_{1}^{U}$. Since $\Gamma$ is poly-torsion-freeabelian, the group ring $\mathbb{Z} \Gamma$ is a right Ore domain and $\mathbb{Q} \Gamma$ embeds into its classical right ring of quotients $\mathcal{K}_{\Gamma}$ [4, Proposition 2.5].

Let $K$ be a knot and $\phi: \pi_{1}\left(M_{K}\right) \rightarrow \Gamma$ a homomorphism. Then one can define the von Neumann $\rho$-invariant $\rho\left(M_{K}, \phi\right) \in \mathbb{R}$ associated with $\phi$ which was introduced by Cheeger and Gromov [3]. When $M_{K}$ bounds an oriented compact 4-manifold $W$ with a homomorphism $\psi: \pi_{1}(W) \rightarrow \Gamma$ extending $\phi$ (i.e., $\left(M_{K}, \phi\right)=\partial(W, \psi)$ ), the von Neumann $\rho$-invariant is computed as

$$
\rho\left(M_{K}, \phi\right)=\sigma_{\Gamma}^{(2)}(W, \psi)-\sigma_{0}(W),
$$

where $\sigma_{\Gamma}^{(2)}(W, \psi)$ is the $L^{2}$-signature of the intersection form on $H_{2}(W ; \mathcal{U} \Gamma)$ and $\sigma_{0}(W)$ is the ordinary signature of $W$. Here $\mathcal{U} \Gamma$ is the algebra of (unbounded) operators affiliated to the von Neumann algebra $\mathcal{N} \Gamma$ of the group $\Gamma$. Often we simply denote $\rho\left(M_{K}, \phi\right)$ by $\rho(K, \phi)$. We refer the reader to [4, Section 5] for more discussion of $L^{2}$-signatures. The following theorem gives an obstruction for a knot being (1.5)-solvable. Note that since $\Gamma^{(2)}=0, \Gamma$ is (1)-solvable.

Theorem 2.2 (4, Theorem 4.2]). In the above setting, if $W$ is an (1.5)-solution for $K$, then $\rho(K, \phi)=0$.

In this paper we are interested in the following representations to $\Gamma$. We construct a representation $\phi_{x}: \pi_{1}\left(M_{K}\right) \rightarrow \Gamma$ associated with a given $x \in H_{1}(M ; \Lambda)$ via the Blanchfield linking form in the following manner. Recall that there is a nonsingular form called the Blanchfield linking form:

$$
B \ell: H_{1}\left(M_{K} ; \Lambda\right) \times H_{1}\left(M_{K} ; \Lambda\right) \rightarrow \mathbb{Q}(t) / \Lambda .
$$

Let $\mu$ be a meridian of $K$ which normally generates $\pi_{1}\left(M_{K}\right)$ and $\epsilon: \pi_{1}\left(M_{K}\right) \rightarrow \mathbb{Z}$, the abelianization sending $\mu$ to 1 . 
Definition 2.3. The representation $\phi_{x}: \pi_{1}\left(M_{K}\right) \rightarrow \Gamma$ is defined to be $\phi_{x}(y)=$ $\left(B \ell\left(x, y \mu^{-\epsilon(y)}\right), \epsilon(y)\right)$ for $y \in \pi_{1}\left(M_{K}\right)$, where $y \mu^{-\epsilon(y)}$ denotes its image in $H_{1}\left(M_{K} ; \Lambda\right)$ as an abuse of notation.

For a $\Lambda$-submodule $P$ of $H_{1}\left(M_{K} ; \Lambda\right)$, define

$$
P^{\perp}:=\left\{y \in H_{1}\left(M_{K} ; \Lambda\right) \mid B \ell(x, y)=0 \text { for all } x \in P\right\} .
$$

We say a $\Lambda$-submodule $P$ of $H_{1}\left(M_{K} ; \Lambda\right)$ is self-annihilating if $P=P^{\perp}$.

Theorem 2.4 (4, Theorems 3.6 and 4.4]). Let $W$ be $a(1)$-solution for $K$ and $P:=\operatorname{ker}\left\{H_{1}\left(M_{K} ; \Lambda\right) \rightarrow H_{1}(W ; \Lambda)\right\}$. Then

(1) $P$ is self-annihilating.

(2) $\phi_{x}$ extends to $\pi_{1}(W)$ if and only if $x \in P$.

\section{Polynomial Splitting theorem}

The following is a stronger form of Theorem 1.1. For two knots $K_{1}$ and $K_{2}$, note that the Alexander module of $K_{1} \# K_{2}$ is isomorphic with the direct sum of the Alexander modules of $K_{1}$ and $K_{2}$.

Theorem 3.1. Let $K_{1}$ and $K_{2}$ be knots and let $M_{1}, M_{2}$, and $M$ be zero surgeries on $K_{1}, K_{2}$, and $K_{1} \# K_{2}$, respectively. Suppose that the Alexander polynomials $\Delta_{K_{1}}(t)$ and $\Delta_{K_{2}}(t)$ are coprime. If there exists a self-annihilating submodule $P$ of $H_{1}(M ; \Lambda)$ with respect to $B \ell$ such that $\rho\left(K_{1} \# K_{2}, \phi_{x}\right)=0$ for all $x \in P$, then there are self-annihilating submodules $P_{i}$ of $H_{1}\left(M_{i} ; \Lambda\right), i=1,2$, such that $P=P_{1} \oplus P_{2}$ and $\rho\left(K_{i}, \phi_{x_{i}}\right)=0$ for all $x_{i} \in P_{i}, i=1,2$.

Proof. First, we show that $P=P_{1} \oplus P_{2}$ for some $\Lambda$-submodules $P_{i} \subset H_{1}\left(M_{i} ; \Lambda\right), i=$ 1,2. To show this, we follow the proof of [10, Lemma 3.1]. Note that $H_{1}(M ; \Lambda)=$ $H_{1}\left(M_{1} ; \Lambda\right) \oplus H_{1}\left(M_{2} ; \Lambda\right)$ and $B \ell=B \ell_{1} \oplus B \ell_{2}$, where $B \ell_{1}$ and $B \ell_{2}$ denote the Blanchfield linking forms of $M_{1}$ and $M_{2}$, respectively. We write an element $z \in$ $H_{1}(M ; \Lambda)$ as $(x, y) \in H_{1}\left(M_{1} ; \Lambda\right) \oplus H_{1}\left(M_{2} ; \Lambda\right)$. Let

$$
P_{1}:=\left\{x \in H_{1}\left(M_{1} ; \Lambda\right) \mid(x, 0) \in P\right\} \text { and } P_{2}:=\left\{y \in H_{1}\left(M_{2} ; \Lambda\right) \mid(0, y) \in P\right\} .
$$

Clearly $P_{1} \oplus P_{2} \subset P$. Conversely, we show $P \subset P_{1} \oplus P_{2}$. For simplicity, denote $\Delta_{K_{i}}(t)$ by $\Delta_{i}$ for $i=1,2$. Since $\Delta_{1}$ and $\Delta_{2}$ are coprime, they are also coprime in $\Lambda$, and hence there are $f$ and $g$ in $\Lambda$ such that $f \Delta_{1}+g \Delta_{2}=1$ in $\Lambda$. Let $z=(x, y) \in P$. Since each $\Delta_{i}$ annihilates $H_{1}\left(M_{i} ; \Lambda\right), \Delta_{1} x=0$ and $\Delta_{2} y=0$. Thus,

$$
\begin{aligned}
& f \Delta_{1} z=\left(f \Delta_{1} x, f \Delta_{1} y\right)=\left(0, f \Delta_{1} y\right), \\
& g \Delta_{2} z=\left(g \Delta_{2} x, g \Delta_{2} y\right)=\left(g \Delta_{2} x, 0\right) .
\end{aligned}
$$

On the other hand,

$$
\begin{aligned}
& x=1 x=f \Delta_{1} x+g \Delta_{2} x=g \Delta_{2} x \\
& y=1 y=f \Delta_{1} y+g \Delta_{2} y=f \Delta_{1} y .
\end{aligned}
$$

Thus we have

$$
\begin{aligned}
& (0, y)=\left(0, f \Delta_{1} y\right)=f \Delta_{1} z, \\
& (x, 0)=\left(g \Delta_{2} x, 0\right)=g \Delta_{2} z .
\end{aligned}
$$

Since $P$ is a $\Lambda$-submodule, we conclude that $(x, 0)$ and $(0, y)$ are in $P$ and hence $x \in P_{1}$ and $y \in P_{2}$. Then $z=(x, y) \in P_{1} \oplus P_{2}$. 


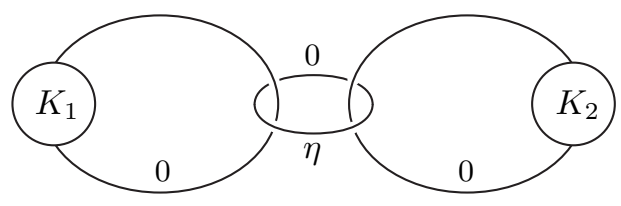

Figure 1

Next, we will show that each $P_{i}$ is self-annihilating with respect to $B \ell_{i}$, i.e., $P_{i}=P_{i}^{\perp}$. For any $x_{1}, x_{2} \in P_{1}$, we have $\left(x_{1}, 0\right),\left(x_{2}, 0\right) \in P$; hence

$$
B \ell_{1}\left(x_{1}, x_{2}\right)=B \ell_{1}\left(x_{1}, x_{2}\right)+B \ell_{2}(0,0)=B \ell\left(\left(x_{1}, 0\right),\left(x_{2}, 0\right)\right)=0 .
$$

Thus, $P_{1} \subset P_{1}^{\perp}$. Conversely, let $x \in P_{1}^{\perp}$. For any $z \in P$, we can write $z=\left(x^{\prime}, y\right)$ for some $x^{\prime} \in P_{1}, y \in P_{2}$. Since $x \in P_{1}^{\perp}, B \ell_{1}\left(x, x^{\prime}\right)=0$ and

$$
B \ell\left((x, 0),\left(x^{\prime}, y\right)\right)=B \ell_{1}\left(x, x^{\prime}\right)+B \ell_{2}(0, y)=0 .
$$

Thus, $(x, 0) \in P^{\perp}$. Since $P=P^{\perp},(x, 0) \in P$ and hence $x \in P_{1}$. Thus, we see that $P_{1}=P_{1}^{\perp}$. Similarly, we can see that $P_{2}=P_{2}^{\perp}$.

Finally, we will show that $\rho\left(K_{i}, \phi_{x_{i}}\right)=0$ for all $x_{i} \in P_{i}, i=1,2$. We construct a cobordism $C$ between the disjoint union $M_{1} \cup M_{2}$ and $M$. Though this construction is well-known, it is an essential step here and is briefly described below. (For details, refer to [5], Section 4].) Attach a 1-handle at the top level between $M_{1} \times[0,1]$ and $M_{2} \times[0,1]$ so that the upper boundary is the zero surgery on the split link $K_{1} \cup K_{2}$. Then attach a 2 -handle along $\eta$ with zero framing as indicated in Figure 1. The resulting $4-$ manifold is the desired $C$. Its boundary at the bottom, $\partial_{-} C$, is the disjoint union $-\left(M_{1} \cup M_{2}\right)$. To see that $\partial_{+} C=M$, slide the 2-handle attached along $K_{2}$ over the 2 -handle attached along $K_{1}$ to get a 2 -handle attached along $K_{1} \# K_{2}$. After the sliding one obtains a surgery diagram where $\eta$ is a meridian of $K_{1}$ and is unlinked from $K_{1} \# K_{2}$. Using $\eta$ one can unlink and unknot $K_{1}$, and therefore one can see that the zero surgery on $\eta \cup K_{1}$ is homeomorphic with the zero surgery on the Hopf link which is homeomorphic with $S^{3}$. Thus $K_{1}$ and $\eta$ can be discarded. (Or one can use the slam-dunk move in [8, p. 163].) The result is a surgery diagram for $M$, and this shows that $\partial_{+} C=M$.

Let $x \in P_{1}$. Then $(x, 0) \in P$. We obtain the representations $\phi_{x}: \pi_{1}\left(M_{1}\right) \rightarrow \Gamma$ and $\phi_{(x, 0)}: \pi_{1}(M) \rightarrow \Gamma$ as defined in Definition 2.3. From the above construction, it is easy to see that $\pi_{1}(C)=\pi_{1}\left(M_{1}\right) * \pi_{1}\left(M_{2}\right) /\left\langle\mu_{1} \mu_{2}^{-1}\right\rangle$, where $*,\langle\rangle$, and $\mu_{i}$ stand for the free product, the subgroup normally generated by the given elements, and the meridian of $K_{i}$ at the point that $\eta$ goes over, respectively. Also, we see that $\pi_{1}(M)=\pi_{1}\left(E_{1}\right) * \pi_{1}\left(E_{2}\right) /\left\langle\mu_{1} \mu_{2}^{-1}, l_{1} l_{2}\right\rangle$, where each $E_{i}$ is the exterior of $K_{i}$ in $S^{3}$ and each $l_{i}$ is the longitude of $K_{i}$. Since $\pi_{1}(C)=\pi_{1}(M) /\left\langle l_{1}\right\rangle=\pi_{1}(M) /\left\langle l_{2}\right\rangle$ and $\phi_{(x, 0)}\left(l_{1}\right)=\phi_{(x, 0)}\left(l_{2}\right)=0$, the representation $\phi_{(x, 0)}$ extends over $\pi_{1}(C)$; i.e., there is a homomorphism $\tilde{\phi}: \pi_{1}(C) \rightarrow \Gamma$ such that $\phi_{(x, 0)}=\tilde{\phi} \circ i_{*}$, where $i_{*}: \pi_{1}(M) \rightarrow \pi_{1}(C)$ is the homomorphism induced by the inclusion $i: M \rightarrow C$. From the construction of $C$, it immediately follows that $\left.\tilde{\phi}\right|_{\pi_{1}\left(M_{1}\right)}=\phi_{x}$ and $\left.\tilde{\phi}\right|_{\pi_{1}\left(M_{2}\right)}$ is the zero map. 
By [5, Lemma 4.2] $H_{*}\left(C ; \mathcal{K}_{\Gamma}\right)=0$, and one can easily show that the inclusion induced map $i_{*}: H_{2}(\partial C ; \mathbb{Z}) \rightarrow H_{2}(C ; \mathbb{Z})$ is surjective. Therefore $\sigma_{\Gamma}^{(2)}(C, \tilde{\phi})=0$ and $\sigma_{0}(C)=0$. Hence

$$
\begin{aligned}
\rho\left(K_{1} \# K_{2}, \phi_{(x, 0)}\right) & =\rho\left(K_{1},\left.\tilde{\phi}\right|_{\pi_{1}\left(M_{1}\right)}\right)+\rho\left(K_{2},\left.\tilde{\phi}\right|_{\pi_{1}\left(M_{2}\right)}\right) \\
& =\rho\left(K_{1}, \phi_{x}\right)+\rho\left(K_{2}, 0\right) \\
& =\rho\left(K_{1}, \phi_{x}\right) .
\end{aligned}
$$

Here, $\rho\left(K_{2}, 0\right)=0$ follows from [5, Property $\left.(2.5)\right]$. Since $(x, 0) \in P$, the left-hand side is zero by the assumption. Therefore $\rho\left(K_{1}, \phi_{x}\right)=0$ for all $x \in P_{1}$. Similarly, $\rho\left(K_{2}, \phi_{y}\right)=0$ for all $y \in P_{2}$. This completes the proof.

\section{EXAmples}

As an application of Theorem 3.1, we present a new example of an infinite family of knots with vanishing Casson-Gordon invariants which are linearly independent in $\mathcal{C}$. In fact, we will show that they are linearly independent in $\mathcal{F}_{1} / \mathcal{F}_{1.5}$. The authors do not know how to show that these knots are linearly independent in $\mathcal{C}$ without using Theorem 3.1

Let $T$ be the infinite set of positive integers each of which is divisible by three distinct primes. For $k \in T$, let $\Phi_{k}(t)$ be the $k-$ th cyclotomic polynomial. As is well-known (for instance see [11, Section 2] and 2, Chapter 5]), there is a slice knot $K_{k}^{\prime}$ that has the cyclic rational Alexander module $H_{1}\left(M_{K_{k}^{\prime}} ; \Lambda\right)=\Lambda /\left(\Phi_{k}(t)^{2}\right)$. Let $J$ be a knot with Arf invariant zero such that the (averaged) integral of the LevineTristram signature function of $J$ is nonzero. For example, one can take $J$ to be the connected sum of two copies of the trefoil. Denote this integral by $\rho(J)$. Let $\eta_{k}$ be the unknot in the complement of a Seifert surface for $K_{k}^{\prime}$ in $S^{3}$ which represents the homology class generating the rational Alexander module for $K_{k}^{\prime}$ over $\Lambda$, i.e., $H_{1}\left(M_{K_{k}^{\prime}} ; \Lambda\right)=\left(\left[\eta_{k}\right]\right)$. Such $\eta_{k}$ exists since any element in the Alexander module for a knot can be represented by a simple closed curve in the complement of the knot which represents a commutator in the knot group, and we may assume that the simple closed curve is unknotted by crossing changes (cf. [16]).

We construct a knot $K_{k}:=K_{k}^{\prime}\left(\eta_{k}, J\right)$ using the satellite construction (or genetic modification following the terminology in [5]) as follows. Take the union of the exterior of $\eta_{k}$ in $S^{3}$ and the exterior of $J$ in $S^{3}$ along their boundary (which is a torus) such that the meridian (respectively the longitude) of $\eta_{k}$ is identified with the longitude (respectively the meridian) of $J$. The resulting ambient manifold is $S^{3}$ and $K_{k}$ is defined to be the image of $K_{k}^{\prime}$ under this identification. In this case, we say that $K_{k}$ is the satellite of the companion $J$ with the axis $\eta_{k}$ and the pattern $K_{k}^{\prime}$. See [5, Section 3] for more details on this construction.

Since $\eta_{k}$ lies in the complement of a Seifert surface for $K_{k}^{\prime}$, the knots $K_{k}^{\prime}$ and $K_{k}$ have the isomorphic rational Alexander module. In particular each $K_{k}$ has the unique nontrivial proper submodule $\left(\Phi_{k}(t)\right)$. Using this property, in [11, Section 6] the second author showed the following: each $K_{k}, k \in T$, is (1)-solvable but not (1.5)-solvable.

Furthermore, we prove the linear independence of $K_{k}, k \in T$. 
Proposition 4.1. The knots $K_{k}, k \in T$, are linearly independent in $\mathcal{F}_{1} / \mathcal{F}_{1.5}$ and hence in the knot concordance group. Moreover, the concordance invariants by Casson-Gordon [1, Letsche [12] and Friedl [6] vanish on connected sums of copies of $K_{k}, k \in T$.

Proof. As mentioned above, all $K_{k}$ are (1)-solvable. Let $k_{1}, \ldots, k_{l}$ be distinct elements in $T$. Suppose that $K:=a_{1} K_{k_{1}} \# \cdots \# a_{l} K_{k_{l}}$ (all $a_{i}$ are integers) is (1.5)solvable. We only need to show that $a_{i}=0$ for all $i$. Suppose to the contrary that $a_{i} \neq 0$ for some $i$. We may assume that $a_{1}>0$ by replacing $K_{k_{1}}$ by $-K_{k_{1}}$ if necessary. For convenience let us denote $K_{k_{1}}$ by $K_{1}$.

Then there is a self-annihilating submodule $P$ of $H_{1}\left(M_{K} ; \Lambda\right)$ such that $\rho\left(M_{K}, \phi_{z}\right)$ $=0$ for all $z \in P$ by Theorems 2.4 and 2.2. Since $\Phi_{k}(t), k \in T$, are all irreducible and are pairwise coprime, by Theorem 3.1 there exists a self-annihilating submodule $P_{1}$ of $H_{1}\left(M_{a_{1} K_{1}} ; \Lambda\right)$ such that $\rho\left(M_{a_{1} K_{1}}, \phi_{x}\right)=0$ for all $x \in P_{1}$.

Recall that $H_{1}\left(M_{a_{1} K_{1}} ; \Lambda\right)=\bigoplus^{a_{1}} H_{1}\left(M_{K_{1}} ; \Lambda\right)$. Pick a nonzero element $x=$ $\left(x_{1}, \ldots, x_{a_{1}}\right) \in P_{1}$. Suppose that $x_{i} \in\left(\Phi_{k_{1}}(t)\right)$ for all $i=1, \ldots, a_{1}$. We may assume that $x_{1} \neq 0$. For the Blanchfield linking form $B \ell$ of $K_{1}, B \ell\left(\eta_{k_{1}}, x_{i}\right) \neq 0$ if and only if $x_{i} \neq 0$, since $\eta_{k_{1}}$ generates $H_{1}\left(M_{K_{1}} ; \Lambda\right)$ and $B \ell$ is nonsingular. Define $\epsilon_{i}$ to be 1 if $x_{i} \neq 0$ and 0 otherwise, for $1 \leq i \leq a_{1}$. Then

$$
\begin{aligned}
\rho\left(M_{a_{1} K_{1}}, \phi_{x}\right) & =\sum_{i=1}^{a_{1}} \rho\left(M_{K_{1}}, \phi_{x_{i}}\right) \\
& =\sum_{i=1}^{a_{1}} \epsilon_{i} \rho(J) \\
& =\rho(J)+\sum_{i=2}^{a_{1}} \epsilon_{i} \rho(J) \\
& \neq 0,
\end{aligned}
$$

which contradicts Theorem 3.1. The first equality can be shown by constructing a cobordism between the disjoint union of $a_{1}$ copies of $M_{K_{1}}$ and $M_{a_{1} K_{1}}$, as we did in the proof of Theorem 3.1. Here we need to add $\left(a_{1}-1\right)$ 1-handles and the same number of 2-handles to construct the cobordism. The argument is almost the same as that in the proof of Theorem 3.1, and hence we do not repeat it here. The second equality of the above equation follows from the proof of [11, Proposition 6.4] and Properties (2.3), (2.4) and (2.5) in [5. The third equality follows since $x_{1} \neq 0$ and $\epsilon_{1}=1$.

Next, if $x_{i_{0}} \notin\left(\Phi_{k_{1}}(t)\right)$ for some $i_{0}, 1 \leq i_{0} \leq a_{1}$, then since $P_{1}$ is a $\Lambda$-submodule and $\Phi_{k_{1}}(t) x_{i_{0}} \neq 0$ in $H_{1}\left(M_{K_{1}} ; \Lambda\right)$,

$$
\Phi_{k_{1}}(t) x=\left(\Phi_{k_{1}}(t) x_{1}, \ldots, \Phi_{k_{1}}(t) x_{a_{1}}\right)
$$

is a nonzero element in $P_{1}$ satisfying $\Phi_{k_{1}}(t) x_{i} \in\left(\Phi_{k_{1}}(t)\right)$ for all $i$. Now, we are back to the previous case, leading to a contradiction.

The second statement follows since all prime power cyclic branched covers of each $K_{k}$ are homology spheres by [15, Theorem 1.2]. 
There are infinitely many knots with vanishing Arf invariant, say $J_{i}, i \in \mathbb{N}$, such that $\rho\left(J_{i}\right), i \in \mathbb{N}$, are linearly independent over $\mathbb{Z}[\underline{5}$, Proposition 2.6]. Using this the second author [1] showed that for a fixed $k \in T$ the $\operatorname{knots} K_{k}^{i}:=K_{k}^{\prime}\left(\eta_{k}, J_{i}\right), i \in \mathbb{N}$, are linearly independent in $\mathcal{F}_{1} / \mathcal{F}_{1.5}$. Note that the knots in Proposition 4.1 cannot be shown to be linearly independent in this way since they are the satellites of the same companion $J$. This demonstrates that Theorem 3.1 is definitely required to prove Proposition 4.1 .

One can easily prove the following proposition using the ideas in the proof of Proposition 4.1, hence we omit the proof. Note that the family of knots in the following proposition includes our examples of knots in Proposition 4.1 .

Proposition 4.2. The knots $K_{k}^{i}, i \in \mathbb{N}, k \in T$, are linearly independent in $\mathcal{F}_{1} / \mathcal{F}_{1.5}$ and hence in $\mathcal{C}$.

\section{ACKNOWLEDGEMENTS}

The authors thank an anonymous referee for a careful reading of this paper and for helpful comments. This work was supported by the Korea Research Foundation Grant funded by the Korean government (MOEHRD, Basic Research Promotion Fund) (KRF-2007-331-C00034).

\section{REFERENCES}

1. A. J. Casson and C. McA. Gordon, Cobordism of classical knots, A la Recherche de la Topologie Perdue. Progress in Math., vol. 62 (Birkhäuser, 1986), pp. 181-199. MR900252

2. J. C. Cha, The structure of the rational concordance group of knots, Mem. Amer. Math. Soc. 189 (2007), no. 885, x+95 pp. MR2343079

3. J. Cheeger and M. Gromov, Bounds on the von Neumann dimension of $L^{2}$-cohomology and the Gauss-Bonnet theorem for open manifolds, J. Differential Geom. 21 (1985), no. 1, 1-34. MR 806699 (87d:58136)

4. T. D. Cochran, K. E. Orr and P. Teichner, Knot concordance, Whitney towers and $L^{2}-$ signatures, Ann. of Math. (2) 157 (2003), no. 2, 433-519. MR1973052 (2004i:57003)

5. T. D. Cochran, K. E. Orr and P. Teichner, Structure in the classical knot concordance group, Comment. Math. Helv. 79 (2004), no. 1, 105-123. MR2031301 (2004k:57005)

6. S. Friedl, Eta invariants as sliceness obstructions and their relation to Casson-Gordon invariants, Algebr. Geom. Topol. 4 (2004), 893-934. MR2100685(2005j:57016)

7. P. M. Gilmer, Classical knot and link concordance, Comment. Math. Helv. 68 (1993), 1-19. MR.1201199 (94c:57007)

8. R. E. Gompf and A. I. Stipsicz, 4-manifolds and Kirby calculus, Graduate Studies in Mathematics 20. American Mathematical Society, Providence, RI, 1999. MR1707327|(2000h:57038)

9. B. Jiang, A simple proof that the concordance group of algebraically slice knots is infinitely generated, Proc. Amer. Math. Soc. 83 (1981), 189-192. MR620010 (82h:57008)

10. S.-G. Kim, Polynomial splittings of Casson-Gordon invariants, Math. Proc. Cambridge Philos. Soc. 138 (2005), no. 1, 59-78. MR2127228 (2005m:57018)

11. T. Kim, Filtration of the classical knot concordance group and Casson-Gordon invariants, Math. Proc. Cambridge Philos. Soc. 137 (2004), no. 2, 293-306. MR 2092061 (2005f:57014)

12. C. F. Letsche, An obstruction to slicing knots using the eta invariant, Math. Proc. Cambridge Philos. Soc. 128 (2000), no. 2, 301-319. MR.1735303 (2001b:57017)

13. J. Levine, Invariants of knot cobordism, Invent. Math. 8 (1969), 98-110. MR0253348 (40:6563)

14. J. Levine, Knot cobordism groups in codimension two, Comment. Math. Helv. 44 (1969), 229-244. MR0246314 (39:7618)

15. C. Livingston, Seifert forms and concordance, Geom. Topol. 6 (2002), 403-408. MR1928840 (2003f:57019) 
16. D. Rolfsen, Knots and links (second printing), Mathematics Lecture Series, vol. 7 (Publish or Perish, Inc., Houston, Texas, 1976, 1990). MR.1277811 (95c:57018)

17. C. T. C. Wall, Surgery on compact manifolds, Second edition. Edited and with a foreword by A. A. Ranicki. Mathematical Surveys and Monographs, 69. American Mathematical Society, Providence, RI, 1999. MR.1687388 (2000a:57089)

Department of Mathematics and Research Institute for Basic Sciences, Kyung Hee University, Seoul 130-701, Korea

E-mail address: sgkim@khu.ac.kr

$U R L:$ web.khu.ac.kr/ sekim

Department of Mathematics, Konkuk University, Seoul 143-701, Korea

E-mail address: tkim@konkuk.ac.kr

$U R L$ : konkuk.ac.kr/ ${ }^{\sim}$ kim 